\title{
Observación de la reacción tisular del precinto comercial de poliamida empleado como método de ligadura en pedículo renal y uterino en conejos
}

\author{
Observation of tissular reaction of commercial polyamide seal used as a method \\ of bond in renal and uterine pedicle in rabbits \\ R Rovere ${ }^{1 *}$, P Bertone ${ }^{1}$, G Bagnis ${ }^{2}$, R Cocco ${ }^{1}$, O Luján ${ }^{1}$, M Sereno ${ }^{1}$, J Wheeler ${ }^{1}$ \\ ${ }^{1}$ Departamento Clínica Animal, ${ }^{2}$ Departamento Patología Animal. Facultad de Agronomía y Veterinaria. \\ Universidad Nacional de Río Cuarto, Argentina.
}

\begin{abstract}
SUMMARY
Ligation is the most common localized and permanent method for the control of haemorrhages. It has been used for more than 4000 years through the employment of different materials, from linen, silk, sheep intestine or tendon fibers until the current use of synthetic materials, absorbables (polyglactine and polydioxanone) or non-absorbables (polypropilene, polyester and polyamide). Staples and vascular clips can also be employed for permanent haemostasia allowing a good occlusion of the vascular pedicles, but their high cost limits their use in veterinary surgery. In this study, tissular reaction at vascular pedicle level was evaluated in rabbits after using a polyamide seal. Six male rabbits underwent nephrectomy and the pedicle was ligatured with polyamide. The remaining six animals underwent ovariohysterectomy with the placement of a seal at uterine cervix level. The haemostasia provided by the implant was immediate and safe after its application. The macroscopic and microscopic observations of the animals showed that the tissular reaction was minimal. Neither tissular necrosis nor a severe inflammation degree were observed during histophatologic studies; nor signs of infection. The polyamide commercial seal is an easy to apply, safe technique, it also causes minimal tissular reaction and has a low cost, making possible its use in vascular pedicle ligatures at surgical veterinary practices.
\end{abstract}

Palabras clave: cirugía, ligadura, poliamida, precinto.

Key words: surgical, ligature, polyamide, seal.

\section{INTRODUCCION}

Los métodos para controlar las hemorragias quirúrgicas pueden ser realizados en vasos de pequeño calibre por medio de pinzamiento, pinzamiento y torsión o por electrocauterización. En los vasos de mediano a gran calibre puede emplearse ligadura simple, por trasfixión con material de sutura o por coagulación con diatermia (Hickman y Walker 1980, Alexander 1989, Baldwin 1993, Boothe 1993).

Knecht y col (1987) señalan que las ligaduras han sido usadas por más de 4.000 años, a través del empleo de distintos materiales desde el lino, la seda, intestino de oveja o fibras de tendón hasta los actuales materiales sintéticos. También hace referencia a que Ambroise Paré (1510-1590) recomendó el empleo de la ligadura como sistema hemostático, con mayores ventajas que la cauterización.

Las grapas y clips vasculares también pueden ser empleados para la hemostasia permanente, los cuales permiten una buena oclusión de los pedículos vasculares;

Aceptado: 22.08.2006.

* Ruta Nacional 36. Km 601. CP X5804BYA. Río Cuarto. Córdoba. Argentina. Tel/Fax: 54-358-4680280. e-mail: rrovere@ayv. unrc.edu.ar pero lamentablemente su elevado costo limita la utilización en la cirugía veterinaria (Boothe 1993, Bellah 1994). Los clips o broches en forma de V se encuentran disponibles como clips metálicos (acero inoxidable, titanio) o como clips absorbibles (poliglactín 910, polidioxanonaPDS-). Estos se emplean en varios procedimientos quirúrgicos en medicina veterinaria para la ligadura de pedículos vasculares en técnicas como la esplenectomía, nefrectomía, ovariectomía y orquiectomía; también para proveer hemostasia en cirugías laparoscópicas. Son de fácil aplicación en áreas de difícil acceso y de alta resistencia; sin embargo el uso de estos implantes esta limitado a vasos o pedículos de menos de $1,1 \mathrm{~mm}$ de diámetro, a lo que se agrega la relativa inestabilidad del clip en el aplicador y la inseguridad del clip colocado en forma inadecuada en el vaso sanguíneo (Boothe 1993, Bellah 1994, Kolata y col 1999).

Las grapas metálicas se utilizan generalmente para ligaduras en masa en la resección de vísceras huecas y órganos parenquimatosos, aunque no es recomendable su aplicación a vasos importantes que no pueden ser comprimidos a $0,75 \mathrm{~mm}$ de espesor o a $7 \mathrm{~mm}$ de ancho, como, por ejemplo, la ligadura de la arteria esplénica (Bellah 1994). El tamaño de la grapa metálica depende del tamaño y grosor del pedículo a ser resecado, por lo que debe tomarse la precaución que la cantidad de tejido a ser 
engrapado no sea excesiva. La grapa ha sido usada en el útero, donde provee hemostasia inmediata (Boothe 1993). También fue empleada para el cierre del cuello vaginal, donde inicialmente se emplearon grapas metálicas, las que eran causantes de disuria y ocasionalmente infección en la línea de colocación de la grapa, por lo que posteriormente se aplicaron grapas de copolímeros (ácido poliglicólico) que se reabsorben en 180 días. Estas grapas absorbibles no disminuyen la contaminación de la cavidad peritoneal, pero se asocian con un mayor grado de inflamación, necrosis tisular y formación de adherencias (Mc Neeley y col 1988).

La ligadura constituye el método más corriente para el control local y permanente de las hemorragias. Los materiales empleados para las ligaduras varían desde los absorbibles (catgut, ac. poliglicólico y PDS) a los no absorbibles (seda, polipropileno, poliéster y nylon).

La poliamida o nylon pertenece a la familia de los termoplásticos semicristalinos, sintetizados a partir de la hexametilentetramina y ácidos-alifáticos en cadena lineal. Es un polímero bastante elástico con buenas propiedades de protección, fatiga elevada y buena resistencia a la abrasión. Es resistente a los aceites, grasas, solventes y álcalis, pero no a los ácidos que los hidrolizan.

Cada nylon particular se describe por uno o dos números, por ejemplo, nylon 6 o nylon 6,6, donde cada número indica la cantidad de átomos de carbono en los monómeros aminados y ácidos (Boothe 1993, Greer y Pearson 1993).

El nylon monofilamento es biológicamente inerte, no tiene capilaridad y causa mínima reacción tisular (Hickman y Walker 1980, Knecht y col 1987, Baker 1990, Boothe 1993, Bellah 1994, Greer y Pearson 1993, Boothe 1998). Al respecto se debe considerar que el concepto de inercia es hoy cuestionable, ya que no existe material sintético (implante) que no interactúe con los elementos líquidos o sólidos del cuerpo y sólo existe una tolerancia, por parte del organismo, al material implantado (Klee y Höcker 2000). Estas características de escasa reactividad han sido ampliamente estudiadas, en Medicina Humana como en Veterinaria. Así se ha demostrado que el nylon conjuntamente con el acero causa mínima inflamación respecto de la seda, cuando se los evaluó histológicamente como sutura en la línea alba de caninos (Wood y col 1984). Presenta menor reacción que la fibra de carbono cuando es utilizado en la reparación del tendón flexor digital en equino (Nixon y col 1984) y demostró ser un sustituto del polipropileno de menos costo, cuando se lo empleó en laparostomías en pacientes humanos (Shah y col 1996). Asimismo se obtuvieron datos idénticos en el proceso de cicatrización y calidad de anastomosis, cuando se lo evaluó en comparación con la polidioxanona (Thomson y col 1995). La incidencia de infección en tejidos contaminados conteniendo nylon monofilamento es inferior que cualquier otra sutura no absorbible, a excepción del polipropileno, en esos mis- mos tejidos; esto, podría deberse a la presencia de metabolitos de la degradación de este material de sutura (Boothe 1993). La reacción microscópica al nylon en heridas quirúrgicas infectadas es mínima comparada con el acero (Boothe 1993).

El principal inconveniente que se menciona respecto del uso del nylon como material de sutura es su escasa maleabilidad e inseguridad en los nudos, por lo que debería evitarse en la ligadura de vasos sanguíneos (Hickman y Walker 1980, Knecht y col 1987, Boothe 1993, Greer y Pearson 1993, Boothe, 1998, Huber y col 1999).

En el mercado existen precintos plásticos, conocidos también como bridas, abrazaderas o prensa cables. Construidos en nylon 6,6 , estos dispositivos se utilizan como método de fijación de diversos elementos, tales como cables, sogas y maderas, siendo también empleados como sistema de seguridad, a efectos de otorgar calidad de inviolable al elemento que se precinta.

Este tipo de precintos ajustables de nylon puede ser empleado para lograr la hemostasia permanente con la correcta oclusión de los pedículos vasculares, con la ventaja, además de su bajo costo, de ser colocado en forma rápida y no requerir de un dispositivo especial de aplicación.

Se propone la utilización de este método de ligadura como una alternativa, para ser aplicada en pedículos vasculares en intervenciones quirúrgicas de la práctica veterinaria.

El objetivo del presente trabajo es evaluar la respuesta tisular provocada en los pedículos vasculares de mediano a gran calibre, sometidos a ligadura con precintos de poliamida, en los animales intervenidos experimentalmente.

\section{MATERIAL Y METODOS}

Se utilizaron precintos comerciales ${ }^{1}$ construidos en poliamida de $2,4 \mathrm{~mm}$ de ancho por $100 \mathrm{~mm}$ de largo, disponibles comercialmente para fijación de cables y tuberías. El material empleado corresponde a poliamida 6,6 de color negro. Si bien los precintos se comercializan además en forma natural (sin coloración), se seleccionó el de color negro para poder localizar los implantes colocados dentro de la cavidad abdominal, cuando se realizaran los muestreos para su estudio histológico (figura 1). Los precintos, en grupo de cuatro, se colocaron en bolsas plásticas cerradas para su esterilización química con óxido de etileno.

Se intervinieron quirúrgicamente doce conejos de raza neocelandesa, ajustándose a los lineamientos del Comité de Etica de la Universidad. Seis de ellos eran hembras y seis machos, entre 3 y 3,5 kg y clínicamente sanos. Luego de un ayuno de sólidos de 24 horas y de

\footnotetext{
Marca PRECINTER SRL.
} 
líquidos de 12 horas fueron anestesiados mediante el siguiente protocolo:

Tranquilización: clorhidrato de xilacina, $5 \mathrm{mg} / \mathrm{kg}$ de peso vivo, intramuscular, 15 minutos antes de la cirugía.

Inducción: clorhidrato de ketamina, $30 \mathrm{mg} / \mathrm{kg}$ de peso vivo. Se administra la mitad de la dosis por vía intramuscular y luego se procede a la canalización de la vena marginal de la oreja y se administra el resto de la dosis.

Mantenimiento: clorhidrato de ketamina (a la mitad de la dosis de inducción) y diazepam $(0,4 \mathrm{mg} / \mathrm{kg}$ de peso vivo), por vía endovenosa.

Antibióticos: ceftriazona sódica, $33 \mathrm{mg} / \mathrm{kg}$ cada 24 horas, intramuscular, durante 48 horas postquirúrgicas.

Analgesia: Se empleó meglumina de flunixim, 0,5 $\mathrm{mg} / \mathrm{kg} / \mathrm{cada} 24$ horas, durante 48 horas postquirúrgicas.

En los seis animales machos se realizó la nefrectomía del riñón izquierdo. Mediante laparotomía mediana prerretroumbilical, se accede a la cavidad peritoneal y una vez localizado el riñón se disecó el pedículo vascular junto con el uréter. Se realizó una ligadura con el empleo de un precinto de poliamida, para lo cual se pasó el extremo libre del precinto a través de la traba que dispone el dispositivo, envolviendo completamente el ilio renal. Se ajustó el precinto tomando el extremo del mismo con el portaagujas y reteniendo en sentido contrario la traba con una pinza hemostática hasta conseguir la máxima compresión posible (figura 2). El resto del extremo libre del precinto se cortó con tijera a 1 $\mathrm{mm}$ de la traba. Se procedió al cierre de la laparotomía mediante un plano único por línea alba, con material de sutura de poliamida 3-0.

En las hembras se realizó la técnica de ovariohisterectomía, a través de una laparotomía mediana preumbilical prepúbica. Localizado el útero, se colocó una pinza hemostática a craneal de cada ovario y se procedió a la ligadura, con hilo de poliamida monofilamento 3/0, de la arteria y vena ovárica. A continuación se procedió a la ligadura del cuello uterino en forma conjunta con el paquete vascular colateral, empleando un precinto comercial de poliamida. El mismo se colocó caudal a la pinza hemostática que comprime la porción uterina a extirpar. Las ligaduras se realizaron con la colocación de los precintos en idéntica forma a la descrita para la nefrectomía. Se realizó la síntesis de la pared abdominal en la forma idéntica a la descrita anteriormente.

Durante el postoperatorio los animales permanecieron en jaulas, bajo observación clínica, controlando diariamente la temperatura, frecuencia respiratoria y cardíaca, color de las membranas mucosas y llenado capilar.

Se procedió a la eutanasia y posterior necropsia de los conejos en tres grupos de cuatro animales cada uno, a los 7, 15 y 30 días postquirúrgicos.

Se tomaron las muestras de los tejidos en los que se aplicaron los precintos, es decir, en los pedículos renales y uterinos. Se tomaron las muestras de los pedículos re- nales y uterinos con los precintos colocados, para estudios histológicos. Se conservaron en solución de Bouin y dentro de las 48 horas posteriores se procedió a su conservación en formaldehído al 4\%; previo a su inclusión en parafina, se retiraron los precintos. Los cortes histológicos fueron coloreados con hematoxilina-eosina y examinados con microscopía de luz.

\section{RESULTADOS Y DISCUSION}

Técnica de aplicación. Los dispositivos fueron colocados en forma cómoda y fijados rápidamente. Una vez colocados no se observó desplazamiento de los implantes, verificando que estaban bien ceñidos. En un caso en el que la colocación fue dudosa, se procedió al corte del precinto para dar lugar a la colocación de uno nuevo.

Controles postoperatorios. En la observación clínica diaria, la temperatura, frecuencia respiratoria, frecuencia cardíaca, color de las membranas mucosas y el llenado capilar fueron normales.

Examen macroscópico. En general, tanto en el pedículo renal como el muñón uterino, a los 7 días, no se observaron adherencias ni características patológicas en el tejido que rodea al sitio de colocación del implante. Sólo se observó una leve reacción local con escaso edema y congestión, limitada a la zona del precinto.

A los 15 días los precintos estaban recubiertos por una lámina de tejido conectivo, no observándose signos de edema ni congestión.

A los 30 días los implantes se encontraban completamente encapsulados por el tejido conectivo, siendo dificultosa su localización e identificación dentro de la cavidad peritoneal al momento de la toma de muestra para el estudio histopatológico. El precinto no perdió sus características estructurales, conservando su forma cerrada (memoria) con la que ceñía al pedículo.

Examen microscópico. A los 7 días el pedículo renal mostró el uréter colapsado y la compresión de la arteria, con presencia de hemorragias a nivel del epitelio ureteral. El tejido periureteral presentaba un leve infiltrado eosinofílico concentrado sobre áreas de tejido adiposo, con reacción leve de polimorfonucleares en este mismo tejido. Hacia la periferia se encontraron restos celulares (cariorrexis) y vasos sanguíneos congestivos por constricción del pedículo.

A nivel del muñón uterino se observó una fuerte reacción inflamatoria con abundante tejido necrótico y ruptura de tejido adiposo. Además, aplastamiento celular muscular con degeneración de miofibrillas del miometrio, con marcada reacción inflamatoria peritoneal y necrosis del tejido graso. El tejido conectivo periférico presentó abundantes células macrofágicas y escasos eosinófilos. 
Las muestras tomadas a los 15 días en el pedículo renal presentaron el uréter con su lumen atelectásico con restos de tejido hialino y restos celulares. En torno del precinto se observaron la proliferación de tejido conectivo, leve infiltrado polimorfonuclear y pocas células macrofágicas. La parte proximal del pedículo presentaba neoformación vascular y proliferación fibroblástica (figura 3).

En el pedículo uterino se encontró un importante grado de atrofia del miometrio con reemplazo del mismo por abundante infiltrado de tejido conectivo. Degeneración del epitelio uterino y moderada cantidad de macrófagos. El tejido conectivo y peritoneal se caracterizó por infiltrado inflamatorio leve, compuesto por eosinófilos diseminados hacia la periferia (figura 4).

Las observaciones a los 30 días, en el pedículo renal, se caracterizaron por reacción inflamatoria a nivel del tejido adiposo, con necrosis grasa, saponificación, presencia escasa de macrófagos, linfocitos y plasmocitos, en respuesta al traumatismo del tejido adiposo. Además, neutrófilos en escasa cantidad y eosinófilos alrededor del pedículo.

En el muñón uterino se observó la presencia de abundante tejido fibroblástico difundido en distintas zonas de la muestra, en reemplazo del miometrio. No se observó reacción inflamatoria importante, sin linfocitos ni granulocitos; sin embargo, se siguieron encontrando de moderado a abundantes eosinófilos, concentrados en zonas periféricas del pedículo.

La hemostasia provista por el implante se obtiene en forma inmediata y segura tras su aplicación, al igual que la utilización de grampas y broches descrita por Boothe (1993) y Bellah (1994).

La utilización de precintos, según lo descrito, presenta algunas ventajas respecto al empleo de otros implantes, ya que son de menor costo respecto a los clips vasculares metálicos y de fácil colocación, sin la necesidad de instrumental especial (Boothe 1993, Bellah 1994, Kolata 1998). Sin embargo, la posibilidad de disponer de un dispositivo para su aplicación haría al método aún más rápido.

A diferencia de los broches y grapas, este dispositivo puede aplicarse a pedículos de tamaño importante, sin riesgos de que se suelte o desplace. Sin embargo, debe asegurarse una correcta colocación y ajuste, como se indica para cualquier otro método de ligadura (Boothe 1993, Bellah 1994, Mc Neeley y col 1988). Se considera inapropiada su aplicación en ligadura de vasos pequeños, puesto que la posibilidad de cierre se vería limitada a pedículos de menos de $3 \mathrm{~mm}$.

El inconveniente más destacado que citan muchos autores para la ligadura con poliamida es su escasa maleabilidad e inseguridad en los nudos (Hickman y Walker 1980, Knecht y col 1987, Boothe 1993, Greer y Pearson 1993, Boothe 1998, Huber y col 1999); este hecho deja de tener importancia, puesto que estos precintos disponen de un sistema de traba (cremallera) que hace imposible deshacerlo una vez que ha sido ceñido.

Al estar los dispositivos constituidos por poliamida, causan una mínima reacción tisular o podrían considerarse biológicamente inertes, como describen a este polímero la mayoría de los autores consultados (Hickman y Walker 1980, Nixon y col 1984, Wood y col 1984, Knecht y col 1987, Baker 1990, Boothe 1993, Greer y Pearso 1993, Bellah 1994, Boothe 1998) y con reacciones similares a la polidioxanona (Thomson y col 1995). Las observaciones macroscópicas y los resultados microscópicos en los animales intervenidos muestran que la reacción tisular fue mínima y se condice con las características descritas para la poliamida por esos mismos autores. A diferencia de lo comunicado por Mc Neeley y col (1988), en los estudios histopatológicos no se observaron necrosis tisular ni un importante grado de inflamación, como tampoco signos de infección, lo que podría deberse al efecto inhibitorio del nylon (Boothe 1993). Es de destacar que la contaminación en la zona del implante puede ser favorecida por las características de mayor rugosidad de la superficie del implante (cremallera), más allá de la utilización de técnicas quirúrgicas asépticas, implantes estériles y antibioticoterapia instaurada (Klee y Höcker 2000).

La presencia de eosinófilos podría deberse a la incorporación de algún aditivo presente en el dispositivo para su coloración. La observación de células gigantes a los 15 días corresponde a la reacción por cuerpo extraño y podría considerarse normal, como la de cualquier material que reacciona con el organismo, considerando su presentación en forma dispersa. La neoformación vascular presente a los 15 días demuestra la escasa reacción por parte del organismo y la puesta en marcha de mecanismos de reparación.

Los resultados obtenidos demuestran que los precintos de poliamida se incorporaron al organismo sin signos de rechazo. El nylon monofilamento es biológicamente inerte, no tiene capilaridad y causa mínima reacción tisular (Baker 1990, Bellah 1994, Boothe 1998, Creer y Pearson 1993, Knecht y col 1987, Hickman y Walter 1980). Al respecto se debe considerar que el concepto de material inerte es hoy cuestionable, ya que no existe material (bioimplante) que no interactúe con los elementos líquidos y sólidos del cuerpo, sino sólo existe una tolerancia por parte del organismo al material implantado (Klee y Hocker 2000).

Se considera que el precinto comercial de poliamida, se presenta como una técnica de fácil aplicación, segura, con mínima reacción tisular y de bajo costo, para ser empleado en la ligadura de pedículos vasculares mayores a $3 \mathrm{~mm}$, en las intervenciones quirúrgicas de la práctica veterinaria. 


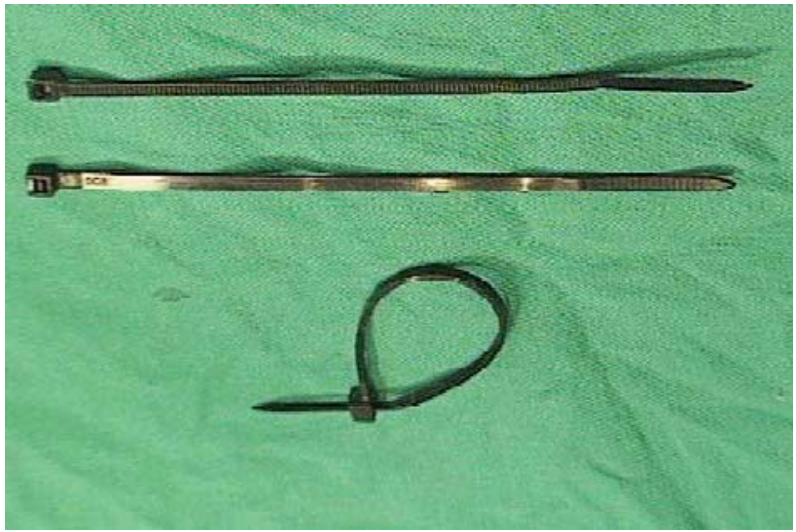

Figura 1. Precinto de nylon en su forma comercial y cerrado a través de la cremallera.

Nylon seal in its commercial form and closed through the rack.

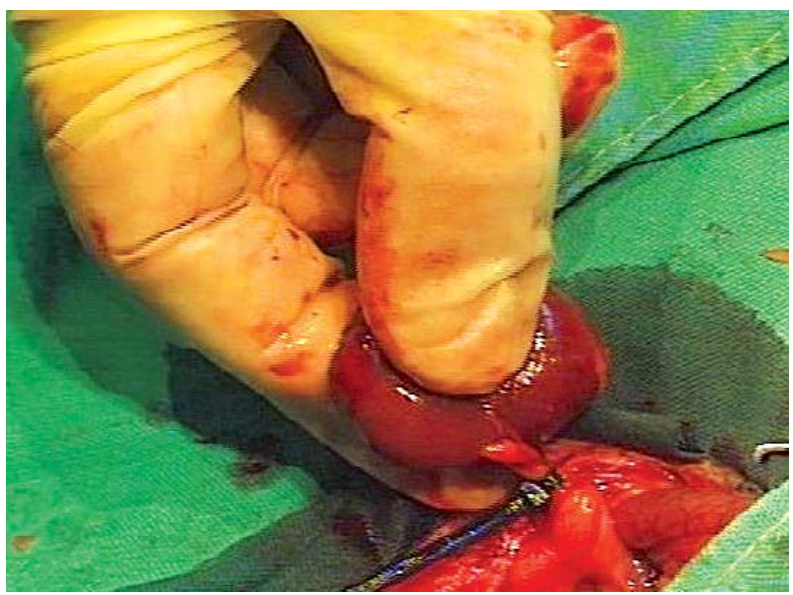

Figura 2. Aplicación del precinto envolviendo el pedículo vascular, durante la nefrectomía. nephrectomy.

Seal application to vascular pedicle during the

\section{RESUMEN}

La ligadura constituye el método más corriente para el control local y permanente de las hemorragias. Ha sido usada por más de 4.000 años a través del empleo de distintos materiales, desde el lino, la seda, intestino de oveja o fibras de tendón, hasta los actuales materiales sintéticos, absorbibles (polioglactin 910 y polidioxanona) o no absorbibles (polipropileno, poliéster y poliamida). Las grapas y clips vasculares pueden también ser empleados para la hemostasia permanente, los cuales permiten una buena oclusión de los pedículos vasculares, pero lamentablemente su elevado costo limita la utilización en la cirugía veterinaria. Se evaluó la respuesta tisular provocada en los pedículos vasculares sometidos a ligadura con precintos de poliamida en conejos. En seis animales machos se realizó la nefrectomía y se ligó el pedículo con un precinto de poliamida; en los seis restantes se procedió a la ovario-histerectomía con la colocación de un precinto a nivel cervical. La hemostasia provista por el implante se obtiene en forma inmediata y segura tras su aplicación. Las observaciones macroscópicas y los resultados microscópicos en los animales intervenidos muestran que la reacción tisular fue mínima. En los estudios histopatológicos no se observaron necrosis tisular ni

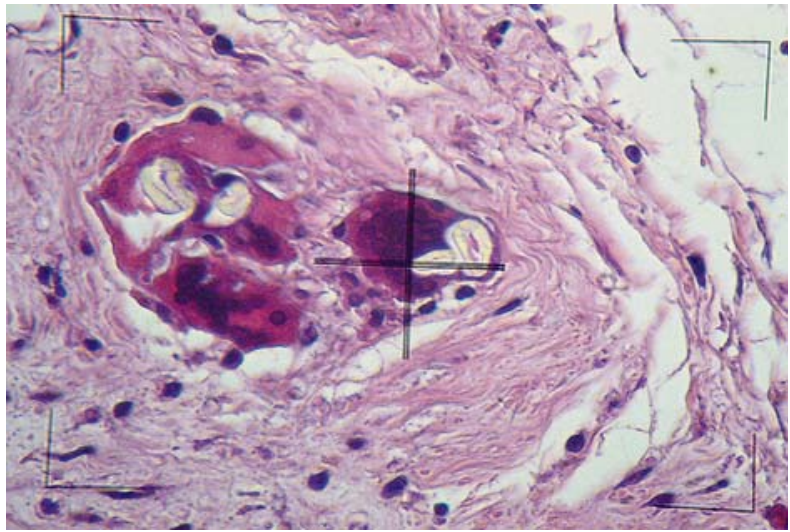

Figura 3. Compresión vascular, con proliferación fibroblástica y presencia de macrófagos en pedículo renal a los 15 días después de la cirugía. H/E $10 x$.

Vascular occlusion with fibroblastic proliferation and macrophagic cells in renal pedicles at day 15 after surgery. H/E 10x.

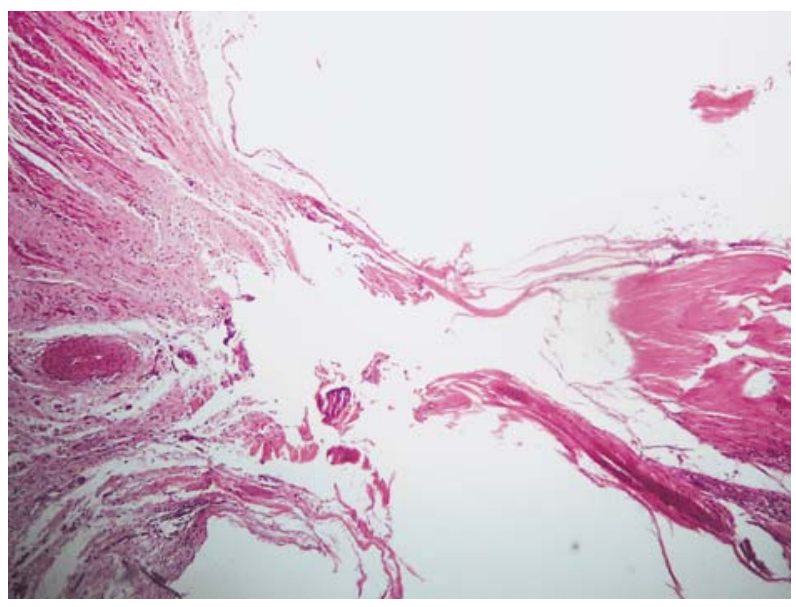

Figura 4. Atrofia del miometrio y endometrio en franca regresión. Sección longitudinal del cuello uterino a los 15 días después de la cirugía $. \mathrm{H} / \mathrm{E}$; $4 \mathrm{x}$.

Miometry and endometry atrophy in regression. Longitudinal section of uterine cervix at day 15 after surgery. H/E; 4x.

un importante grado de inflamación, como tampoco signos de infección. El precinto comercial de poliamida se presenta como una técnica de fácil aplicación, segura, con mínima reacción tisular y de bajo costo para ser empleado en la ligadura de pedículos vasculares en las intervenciones quirúrgicas en la práctica veterinaria.

\section{AGRADECIMIENTOS}

Los autores quieren agradecer a la Sra. Nelsy Schleef, por los trabajos de las muestras histopatológicas y al Sr. Raúl Rodríguez, por el cuidado de los animales.

\section{REFERENCIAS}

Alexander A. 1989. Técnica Quirúrgica en Animales. Interamericana. DF México, México, Pp 88-100.

Baker G J. 1990. Instrumental Quirúrgico. En Bedford P G C. Atlas de Técnicas Quirúrgicas Caninas. Acribia, Zaragoza, España, Pp 12-14. 
Baldwin C J, R J Cowell, M Kostolich, R D Tyer, D C Sempere. 1993. Hemostasis: Physology, Diagnosis and Treatment of Bleeding Disorders in Surgical Patients. En: Slatter D. Textbook of Small Animal Surgery. WB Saunders, Philadelphia, USA, Pp 50-51.

Bellah J R. 1994. Surgical Stapling of the Spleen, Pancreas, Liver, and Urogenital Tract Small Anim Pract 24, 375-383.

Boothe, H W. 1993. Suture Materials, Tisue Adhesives, Staples and Ligation Clips. En Slatter D. Textbook of Small Animal Surgery. WB Saunders, Philadelphia, USA, Pp 206-211.

Boothe H W. 1998. Selecting Suture Materials for Small Animal Surgery. Compend Cont Educ Pract 20: 355-358.

Greer R T, P T Pearson. 1993. Biomaterials. En Slatter D. Textbook of Small Animal Surgery. WB Saunders, Philadelphia, USA, Pp 105-109.

Hickman J, R G Walker. 1980. Principios Generales de la Cirugía. En: Atlas de Cirugía Veterinaria C.E.C.S.A, DF México, México, Pp 28-32.

Huber D J, E L Egger, S P James. 1999. The Effect of Knotting Methods on the Structural Properties of Large Diameter Nonabsorbable Monofilament Sutures. Vet Surg 28, 260-267.

Klee D, H Höcker. 2000. Polimers for Biomedical Apliccations: Improvement of the Interface Compatibility. Adv Polym Sci 149, 1-9.

Knecht C D, A R Allen, D J Williams, J H Johnson. 1987. Suture Materials. En Fundamental Techniques in Veterinary Surgery. WB Saunders, Philadelphia, USA, Pp 28-35.
Kolata J R, L J Freeman.1999. Access, Port Placement and Basic Endosurgical Skill. En Veterinary Endosurgery. Mosby, Missouri, USA, Pp 55-58.

Mc Neeley S G, T E Elkins, D M Portz. 1988. Comparison of copolymer staple vs chronic suture during hysterectomy: Gross, histologic and microbiologic findings. Obst Gynecol 72, 872-865.

Nixon A J, T S Stashak, F W Smith, R W Nordin. 1984. Comparison of carbon fibre and nylon suture for repair of transected flexor tendons in the horse. Equine Vet J 16, 93-102.

Shah S R, A B Abhayankar, S K Mathur. 1996. Meshlaparostomy. A comparison between polypropylene mesh and indigenous nylon net. Trop. Gastroenterol 17, 49-54.

Sicard G K, K Hayashi, P A Manley. 2002. Evaluation of 5 types of fishing material, 2 sterilization methods and a crimp-clamp system for extra articular stabilization of the canine stifle joint. Vet Surg 31, 78-84.

Thomson S R, M A Gregory, M Mars, J Natasen, T Naickir, L W Baker. 1995. Morphological aspects of microarterial anastomoses: a comparison of nylon with polydioxanone. $\mathrm{Br} J$ Plast Surg 48,165-171.

Wood D S, J E Collins, R Wallshaw.1984. Tissue reaction to nonabsorbable suturematerial in the canine linea alba: a histological evaluation. J Am Anim Hosp Assoc 20, 39-44. 